Article

\title{
On the Difference of Inverse Coefficients of Univalent Functions
}

\author{
Young Jae $\operatorname{Sim}^{1, *(\mathbb{D}) \text { and Derek Keith Thomas }}{ }^{2}$ \\ 1 Department of Mathematics, Kyungsung University, Busan 48434, Korea \\ 2 Department of Mathematics, Swansea University, Bay Campus, Swansea SA1 8EN, UK; \\ d.k.thomas@swansea.ac.uk \\ * Correspondence: yjsim@ks.ac.kr
}

Received: 20 November 2020; Accepted: 8 December 2020; Published: 9 December 2020

Abstract: Let $f$ be analytic in the unit disk $\mathbb{D}=\{z \in \mathbb{C}:|z|<1\}$, and $\mathcal{S}$ be the subclass of normalized univalent functions with $f(0)=0$, and $f^{\prime}(0)=1$. Let $F$ be the inverse function of $f$, given by $F(z)=\omega+\sum_{n=2}^{\infty} A_{n} \omega^{n}$ for some $|\omega| \leq r_{0}(f)$. Let $\mathcal{S}^{*} \subset \mathcal{S}$ be the subset of starlike functions in $\mathbb{D}$, and $\mathcal{C}$ the subset of convex functions in $\mathbb{D}$. We show that $-1 \leq\left|A_{3}\right|-\left|A_{2}\right| \leq 3$ for $f \in \mathcal{S}$, the upper bound being sharp, and sharp upper and lower bounds for $\left|A_{3}\right|-\left|A_{2}\right|$ for the more important subclasses of $\mathcal{S}^{*}$ and $\mathcal{C}$, and for some related classes of Bazilevič functions.

Keywords: univalent functions; starlike functions; convex functions; Bazilevič functions; difference of coefficients; inverse coefficients

MSC: 30C45; 30C50

\section{Introduction}

Let $\mathcal{A}$ denote the class of analytic functions $f$ in the unit disk $\mathbb{D}=\{z \in \mathbb{C}:|z|<1\}$ normalized by $f(0)=0=f^{\prime}(0)-1$. Then, for $z \in \mathbb{D}, f \in \mathcal{A}$ has the following representation

$$
f(z)=z+\sum_{n=2}^{\infty} a_{n} z^{n} .
$$

Let $\mathcal{S}$ denote the subclass of all univalent functions in $\mathcal{A}$.

In 1985, de Branges [1] solved the famous Bieberbach conjecture by showing that if $f \in \mathcal{S}$, then $\left|a_{n}\right| \leq n$ for $n \geq 2$, with equality for Koebe function $f(z)=k(z):=z /(1-z)^{2}$ or its rotation. It was, therefore, natural to ask: if for $f \in \mathcal{S}$, the inequality ||$a_{n+1}|-| a_{n}|| \leq 1$ is true when $n \geq 2$ ? This was shown not to be the case even when $n=2$ [2], and that the following sharp bounds hold:

$$
-1 \leq\left|a_{3}\right|-\left|a_{2}\right| \leq \frac{3}{4}+e^{-\lambda_{0}}\left(2 e^{-\lambda_{0}}-1\right)=1.029 \cdots
$$

where $\lambda_{0}$ is the unique value of $\lambda$ in $0<\lambda<1$, satisfying the equation $4 \lambda=e^{\lambda}$.

Hayman [3] showed that if $f \in \mathcal{S}$, then ||$a_{n+1}|-| a_{n}|| \leq C$, where $C$ is an absolute constant. The exact value of $C$ is unknown, the best estimate to date being $C=3.61 \cdots$ [4], which because of the sharp estimate above when $n=2$, cannot be reduced to 1 .

Hayman's seminal result ||$a_{n+1}|-| a_{n}|| \leq C$ for $n \geq 2$, was proved in 1963, using his distinctive method developed to study areally mean $p$-valent functions [5] (Chapter 6). A different proof was provided by Milin, using the now well-known Lebedev-Milin inequalities [2] (p. 143), and an excellent account of this result can be found in Duren's book [2]. Little progress has been made estimating the 
value of $C$. It was shown by Ilina [6] in 1968 that $C<4.26 \ldots$, which, using a modification of Milin's method, Grispan [4] improved in 1976 to show that for $n \geq 2$,

$$
-2.97 \cdots<\left|a_{n+1}\right|-\left|a_{n}\right|<3.61 \ldots
$$

No other advances appear to have been made in this direction during the intervening years, until a recent result of Obradović, Thomas and Tuneski [7] who, using the Grunsky inequalities, showed that the upper bound for $C$ can be improved when $n=3$ to $\left|a_{4}\right|-\left|a_{3}\right| \leq 2.1033 \ldots$.

Thus, apart from the inequalities for $n=2$ above, there appears to be no known sharp upper or lower bounds for $\left|a_{n+1}\right|-\left|a_{n}\right|$ when $n \geq 3$ for functions in $\mathcal{S}$.

For $f \in \mathcal{S}$ denote by $F$ the inverse of $f$ given by

$$
F(\omega)=z+\sum_{n=2}^{\infty} A_{n} \omega^{n}
$$

valid on some disk $|\omega| \leq r_{0}(f)$.

Although sharp bounds are known for $\left|A_{n}\right|$ for $n \geq 2$ [8] when $f \in \mathcal{S}$, there appears to be no known bounds for the difference of coefficients $\left|A_{n+1}\right|-\left|A_{n}\right|$, even in the case $n=2$. In Section 4, we provide sharp upper and lower bounds for $\left|A_{3}\right|-\left|A_{2}\right|$.

In [9], the present authors gave sharp upper and lower bounds for $\left|a_{3}\right|-\left|a_{2}\right|$, when $f$ belongs to the most important subclasses of starlike and convex functions in $\mathbb{D}$.

The purpose of subsequent sections is to find corresponding sharp upper and lower bounds for $\left|A_{3}\right|-\left|A_{2}\right|$, when $f$ belongs to the same subclasses of starlike and convex functions considered in [9], and to compare any invariance properties.

We note at this point that since $f\left(f^{-1}(\omega)\right)=\omega$, equating coefficients gives

$$
\begin{aligned}
& A_{2}=-a_{2}, \\
& A_{3}=2 a_{2}^{2}-a_{3} .
\end{aligned}
$$

\section{Definitions}

We define the classes $\mathcal{S}^{*}$ and $\mathcal{C}$ of starlike and convex functions to be the most significant subclasses of $\mathcal{S}^{*}$ and $\mathcal{C}$, which were considered in [9]. We also give definitions of some related Bazilevič functions, which will also be considered in this paper.

Definition 1. Let $f \in \mathcal{A}$ be given by (1). Denote by $\mathcal{S}^{*}$ the subclass of $\mathcal{A}$ consisting of starlike functions, i.e., functions $f$ which map $\mathbb{D}$ onto a set which is star-shaped with respect to the origin. Then, it is well-known that a function $f \in \mathcal{S}^{*}$ if, and only if, for $z \in \mathbb{D}$

$$
\operatorname{Re}\left\{\frac{z f^{\prime}(z)}{f(z)}\right\}>0
$$

We note here that without doubt the most complete solution to finding sharp bounds for the difference of successive coefficients is the following theorem of Leung [10], who in 1978, proved the sharp inequality ||$a_{n+1}|-| a_{n}|| \leq 1$ for $n \geq 2$, when $f \in \mathcal{S}^{*}$, the proof of which relies on a lemma concerning functions of positive real, and the third Lebedev-Milin inequality.

Definition 2. Let $f \in \mathcal{A}$ be given by (1). Denote by $\mathcal{C}$ the subclass of $\mathcal{A}$ consisting of convex functions, i.e., functions $f$ which map $\mathbb{D}$ onto a set which is convex. A function $f \in \mathcal{C}$ if, and only if, for $z \in \mathbb{D}$

$$
\operatorname{Re}\left\{1+\frac{z f^{\prime \prime}(z)}{f^{\prime}(z)}\right\}>0
$$


Thus, $f \in \mathcal{C}$ if, and only if, $z f^{\prime} \in \mathcal{S}^{*}$ for $z \in \mathbb{D}$.

Definition 3. Let $f \in \mathcal{A}$ be given by (1). Denote by $\mathcal{S}^{*}(\alpha)$ the subclass of $\mathcal{S}^{*}$ consisting of starlike functions of order $\alpha$, i.e., for $0 \leq \alpha<1$ and $z \in \mathbb{D}$,

$$
\operatorname{Re}\left\{\frac{z f^{\prime}(z)}{f(z)}\right\}>\alpha
$$

Definition 4. Let $f \in \mathcal{A}$ be given by (1). Denote by $\mathcal{C}(\alpha)$ the subclass of $\mathcal{C}$ consisting of convex functions of order $\alpha$, i.e., for $0 \leq \alpha<1$ and $z \in \mathbb{D}$,

$$
\operatorname{Re}\left\{1+\frac{z f^{\prime \prime}(z)}{f^{\prime}(z)}\right\}>\alpha
$$

Definition 5. Let $f \in \mathcal{A}$ be given by (1). Denote by $\mathcal{S}^{* *}(\alpha)$ the subclass of $\mathcal{S}^{*}$ consisting of strongly starlike functions order $\alpha$, i.e., for $0<\alpha \leq 1$ and $z \in \mathbb{D}$,

$$
\left|\arg \frac{z f^{\prime}(z)}{f(z)}\right| \leq \frac{\alpha \pi}{2}
$$

Definition 6. Let $f \in \mathcal{A}$ be given by (1). Denote by $\mathcal{C}^{* *}(\alpha)$ the subclass of $\mathcal{C}$ consisting of strongly convex functions order $\alpha$, i.e., for $0<\alpha \leq 1$ and $z \in \mathbb{D}$,

$$
\left|\arg \left(1+\frac{z f^{\prime \prime}(z)}{f^{\prime}(z)}\right)\right| \leq \frac{\alpha \pi}{2} .
$$

Definition 7. Let $f \in \mathcal{A}$ be given by (1). Denote by $\mathcal{B}_{1}(\alpha, \beta)$ the subclass of $\mathcal{S}$ consisting of the Bazilevic functions $f$ satisfying

$$
\operatorname{Re}\left\{f^{\prime}(z)\left(\frac{f(z)}{z}\right)^{\beta-1}\right\}>\alpha,
$$

for $0 \leq \alpha<1, \beta \geq 0$, and $z \in \mathbb{D}$

Definition 8. Let $f \in \mathcal{A}$ be given by (1). Denote by $\mathcal{B}_{1}^{*}(\alpha, \beta)$ the subclass of $\mathcal{S}$ consisting of the Bazilevič functions $f$ satisfying

$$
\left|\arg \left\{f^{\prime}(z)\left(\frac{f(z)}{z}\right)^{\beta-1}\right\}\right| \leq \frac{\alpha \pi}{2},
$$

for $0<\alpha \leq 1, \beta \geq 0$, and $z \in \mathbb{D}$.

\section{Preliminary Lemmas}

Denote by $\mathcal{P}$, the class of analytic functions $p$ with a positive real part on $\mathbb{D}$ given by

$$
p(z)=1+\sum_{n=1}^{\infty} p_{n} z^{n}=1+p_{1} z+p_{2} z^{2}+\cdots
$$

We will use the following lemmas for the coefficients of functions $\mathcal{P}$, given by (5).

Lemma 1. [2] (p. 41) For $p \in \mathcal{P},\left|p_{n}\right| \leq 2$ for $n \geq 1$. The inequalities are sharp.

Lemma 2. [11] If $p \in \mathcal{P}$, then

$$
\begin{aligned}
& p_{1}=2 \zeta_{1} \\
& p_{2}=2 \zeta_{1}^{2}+2\left(1-\left|\zeta_{1}\right|^{2}\right) \zeta_{2}
\end{aligned}
$$


for some $\zeta_{i} \in \overline{\mathbb{D}}, i \in\{1,2\}$.

For $\zeta_{1} \in \partial \mathbb{D}$, the boundary of $\mathbb{D}$, there is a unique function $p \in \mathcal{P}$ with $p_{1}$ as above, namely,

$$
p(z)=\frac{1+\zeta_{1} z}{1-\zeta_{1} z} \quad(z \in \mathbb{D}) .
$$

For $\zeta_{1} \in \mathbb{D}$ and $\zeta_{2} \in \partial \mathbb{D}$, there is a unique function $p \in \mathcal{P}$ with $p_{1}$ and $p_{2}$ as above, namely,

$$
p(z)=\frac{1+\left(\bar{\zeta}_{1} \zeta_{2}+\zeta_{1}\right) z+\zeta_{2} z^{2}}{1+\left(\bar{\zeta}_{1} \zeta_{2}-\zeta_{1}\right) z-\zeta_{2} z^{2}} \quad(z \in \mathbb{D}) .
$$

Lemma 3. [12] Suppose that $p \in \mathcal{P}$, with coefficients given by (5), and $p_{1} \geq 0$.

Then, for some complex-valued $y$ with $|y| \leq 1$,

$$
2 p_{2}=p_{1}^{2}+y\left(4-p_{1}^{2}\right)
$$

\section{Inverse Coefficient Differences for $\mathcal{S}$}

Theorem 1. Let $f \in \mathcal{S}$, with coefficients of $F$ given by (2). Then

$$
-1 \leq\left|A_{3}\right|-\left|A_{2}\right| \leq 3 \text {. }
$$

The upper bound is sharp.

Proof. For the upper bound, write

$$
\left|A_{3}\right|-\left|A_{2}\right|=\left|a_{3}-a_{2}^{2}-a_{2}^{2}\right|-\left|a_{2}\right| \leq\left|a_{3}-a_{2}^{2}\right|+\left|a_{2}\right|^{2}-\left|a_{2}\right|,
$$

which on using the classical inequality $\left|a_{3}-a_{2}^{2}\right| \leq 1$ and $\left|a_{2}\right| \leq 2$, gives the required bound.

The lower bound follows from the method in Duren's book [2] (p. 114).

If $\left|A_{2}\right| \leq 1$, then clearly $\left|A_{2}\right|-\left|A_{3}\right| \leq 1$.

If $\left|A_{2}\right| \geq 1$ write

$$
\left|A_{2}\right|-\left|A_{3}\right|=\left|A_{2}\right|^{2}-\left|A_{3}\right|+\left|A_{2}\right|\left(1-\left|A_{2}\right|\right) .
$$

Now, note that $\left|A_{2}\right|^{2}-\left|A_{3}\right| \leq\left|A_{3}-A_{2}^{2}\right|=\left|a_{3}-a_{2}^{2}\right| \leq 1$, and so

$$
\left|A_{2}\right|-\left|A_{3}\right|=\left|A_{2}\right|^{2}-\left|A_{3}\right|+\left|A_{2}\right|\left(1-\left|A_{2}\right|\right) \leq 1 .
$$

The upper bound is clearly sharp when $f$ is the Koebe function. Whether the lower bound -1 is the best possible is an open problem.

Note, that we will see later that when $f \in \mathcal{S}^{*}$,

$$
-\sqrt{\frac{2}{3}} \leq\left|A_{3}\right|-\left|A_{2}\right| \leq 3
$$

In the subsequent sections, we note at the outset that all of the classes of functions considered, and the functional $\left|A_{3}\right|-\left|A_{2}\right|$ are rotationally invariant.

\section{Inverse Coefficient Differences for Subclasses of $\mathcal{S}$}

In this section, we will find the sharp upper and lower bounds of inverse coefficient differences for $\mathcal{S}^{*}(\alpha), \mathcal{S}^{* *}(\alpha), \mathcal{C}(\alpha), \mathcal{C}^{* *}(\alpha), \mathcal{B}_{1}(\alpha, \beta)$, and $\mathcal{B}_{1}^{*}(\alpha, \beta)$. We first present a proposition which gives a general method of proof applicable to all the subsequent classes considered, noting that individual proofs of all the theorems that follow can also be obtained using the same methodology. 
Proposition 1. Let $B_{1}, B_{2}$ and $B_{3}$ be numbers such that $B_{1} \geq 0, B_{2} \in \mathbb{C}$, and $B_{3} \in \mathbb{R}$. Let $p \in \mathcal{P}$ be of the form (5).

Define $\Psi_{+}\left(p_{1}, p_{2}\right)$ and $\Psi_{-}\left(p_{1}, p_{2}\right)$ by

$$
\Psi_{+}\left(p_{1}, p_{2}\right)=\left|B_{2} p_{1}^{2}+B_{3} p_{2}\right|-\left|B_{1} p_{1}\right|
$$

and $\Psi_{-}\left(p_{1}, p_{2}\right)=-\Psi_{+}\left(p_{1}, p_{2}\right)$. Then

$$
\Psi_{+}\left(p_{1}, p_{2}\right) \leq \begin{cases}\left|4 B_{2}+2 B_{3}\right|-2 B_{1}, & \text { when }\left|2 B_{2}+B_{3}\right| \geq\left|B_{3}\right|+B_{1} \\ 2\left|B_{3}\right|, & \text { otherwise, }\end{cases}
$$

and

$$
\Psi_{-}\left(p_{1}, p_{2}\right) \leq \begin{cases}2 B_{1}-B_{4}, & \text { when } B_{1} \geq B_{4}+2\left|B_{3}\right| \\ 2 B_{1} \sqrt{\frac{2\left|B_{3}\right|}{B_{4}+2\left|B_{3}\right|}}, & \text { when } B_{1}^{2} \leq 2\left|B_{3}\right|\left(B_{4}+2\left|B_{3}\right|\right) \\ 2\left|B_{3}\right|+\frac{B_{1}^{2}}{B_{4}+2\left|B_{3}\right|}, & \text { otherwise, }\end{cases}
$$

where $B_{4}=\left|4 B_{2}+2 B_{3}\right|$. All inequalities in (6) and (7) are sharp.

Proof. First, assume that $B_{2}=B_{3}=0$. Then, we can easily check (6) and (7) hold. Indeed, we have

$$
\Psi_{+}\left(p_{1}, p_{2}\right) \leq-\left|B_{1} p_{1}\right| \leq 0=2\left|B_{3}\right|
$$

and

$$
\Psi_{-}\left(p_{1}, p_{2}\right) \leq\left|B_{1} p_{1}\right| \leq 2 B_{1}=2 B_{1}-B_{4} .
$$

Next, we assume that $B_{2} \neq 0$ or $B_{3} \neq 0$ so that $B_{4}+2\left|B_{3}\right|>0$. Since $\mathcal{P}$ and $\Psi_{+}\left(p_{1}, p_{2}\right)$ are rotationally invariant, using Lemma 1 , we may assume that $p_{1}=p$ with $0 \leq p \leq 2$. Using Lemma 3 and the fact that $|y| \leq 1$, we obtain

$$
\Psi_{+}\left(p_{1}, p_{2}\right) \leq B_{5} p^{2}-B_{1} p+2\left|B_{3}\right|=: g(p),
$$

where $B_{5}=\left(\left|2 B_{2}+B_{3}\right|-\left|B_{3}\right|\right) / 2$.

If $\left|2 B_{2}+B_{3}\right| \geq\left|B_{3}\right|+B_{1}$, then $B_{5} \geq 0$. Since $g$ is convex on $[0,2]$, we have

$$
\Psi_{+}\left(p_{1}, p_{2}\right) \leq g(p) \leq \max \{g(0), g(2)\}=4 B_{5}-2 B_{1}+2\left|B_{3}\right|, \quad p \in[0,2] .
$$

Assume now that $\left|2 B_{2}+B_{3}\right|<\left|B_{3}\right|+B_{1}$. If $B_{5} \geq 0$, then

$$
\Psi_{+}\left(p_{1}, p_{2}\right) \leq g(p) \leq \max \{g(0), g(2)\}=2\left|B_{3}\right|, \quad p \in[0,2]
$$

If $B_{5} \leq 0$, then $g$ is decreasing on $[0,2]$. Hence, $\Psi_{+}\left(p_{1}, p_{2}\right) \leq g(0)=2\left|B_{3}\right|$, which gives (6).

Equality in (6) holds for $p(z)=(1+z) /(1-z)$ when $\left|2 B_{2}+B_{3}\right| \geq\left|B_{3}\right|+B_{1}$, and equality in (6) holds for $p(z)=\left(1+z^{2}\right) /\left(1-z^{2}\right)$ when $\left|2 B_{2}+B_{3}\right|<\left|B_{3}\right|+B_{1}$.

For (7), we use Lemma 2, noting that if $B_{1}=0$, the inequality is obvious. After a rotation so that $\zeta_{1}=t$, where $0 \leq t \leq 1$, and writing $\zeta_{2}=s e^{i \phi}$ with $0 \leq s \leq 1$ and $0 \leq \phi \leq 2 \pi$, we obtain

$$
\begin{aligned}
\Psi_{-}\left(p_{1}, p_{2}\right) & =2 B_{1} t-\left|\left(4 B_{2}+2 B_{3}\right) t^{2}+2 B_{3}\left(1-t^{2}\right) s e^{i \phi}\right| \\
& \leq 2 B_{1} t-\left|B_{4} t^{2}-2\right| B_{3}\left|\left(1-t^{2}\right) s\right|=: \Psi(t, s) .
\end{aligned}
$$


Let

$$
t_{0}=\sqrt{\frac{2\left|B_{3}\right|}{B_{4}+2\left|B_{3}\right|}}
$$

First, we assume that $0 \leq t \leq t_{0}$. Then

$$
\Psi(t, s) \leq 2 B_{1} t \leq 2 B_{1} t_{0}=2 B_{1} \sqrt{\frac{2\left|B_{3}\right|}{B_{4}+2\left|B_{3}\right|}}, \quad t \in\left[0, t_{0}\right], s \in[0,1] .
$$

Next, we assume that $t_{0} \leq t \leq 1$. Then, $B_{4} t^{2}-2\left|B_{3}\right|\left(1-t^{2}\right) s \geq 0$ for $s \in[0,1]$, since

$$
\begin{aligned}
B_{4} t^{2}-2\left|B_{3}\right|\left(1-t^{2}\right) s & =\left(B_{4}+2\left|B_{3}\right| s\right) t^{2}-2\left|B_{3}\right| s \geq\left(B_{4}+2\left|B_{3}\right| s\right) t_{0}^{2}-2\left|B_{3}\right| s \\
& =\frac{2\left|B_{3}\right| B_{4}(1-s)}{B_{4}+2\left|B_{3}\right|} \geq 0, \quad t \in\left[t_{0}, 1\right], s \in[0,1] .
\end{aligned}
$$

Thus, we have

$$
\begin{aligned}
\Psi(t, s) & =2 B_{1} t-B_{4} t^{2}+2\left|B_{3}\right|\left(1-t^{2}\right) s \\
& \leq-\left(B_{4}+2\left|B_{3}\right|\right) t^{2}+2 B_{1} t+2\left|B_{3}\right|=: h(t), \quad t \in\left[t_{0}, 1\right], s \in[0,1] .
\end{aligned}
$$

(a) If $B_{1} \geq B_{4}+2\left|B_{3}\right|$, then $h$ is increasing on $\left[t_{0}, 1\right]$, since

$$
h^{\prime}(t)=-2\left(B_{4}+2\left|B_{3}\right|\right) t+2 B_{1} \geq-2\left(B_{4}+2\left|B_{3}\right|\right)+2 B_{1} \geq 0, \quad t \in\left[t_{0}, 1\right] .
$$

Thus, it follows from (10) that

$$
\Psi(t, s) \leq h(1)=2 B_{1}-B_{4}, \quad t \in\left[t_{0}, 1\right], s \in[0,1] .
$$

(b) If $B_{1}^{2} \leq 2\left|B_{3}\right|\left(B_{4}+2\left|B_{3}\right|\right)$, then $h$ is decreasing on $\left[t_{0}, 1\right]$, since

$$
\begin{aligned}
h^{\prime}(t) & =-2\left(B_{4}+2\left|B_{3}\right|\right) t+2 B_{1} \\
& \leq-2\left(B_{4}+2\left|B_{3}\right|\right) t_{0}+2 B_{1} \\
& =-2 \sqrt{2\left|B_{3}\right|\left(B_{4}+2\left|B_{3}\right|\right)}+2 B_{1} \leq 0,
\end{aligned}
$$

when $t \in\left[t_{0}, 1\right]$. Thus, by (10)

$$
\Psi(t, s) \leq h\left(t_{0}\right)=2 B_{1} \sqrt{\frac{2\left|B_{3}\right|}{B_{4}+2\left|B_{3}\right|}}, \quad t \in\left[t_{0}, 1\right], s \in[0,1] .
$$

(c) When $B_{1}<B_{4}+2\left|B_{3}\right|$ and $B_{1}^{2}>2\left|B_{3}\right|\left(B_{4}+2\left|B_{3}\right|\right)$, we note that

$$
t_{0}<\frac{B_{1}}{B_{4}+2\left|B_{3}\right|}=: t_{1}<1,
$$

and $h^{\prime}(t)=0$ when $t=t_{1}$. Therefore, from (10), we have

$$
\Psi(t, s) \leq h\left(t_{1}\right)=2\left|B_{3}\right|+\frac{B_{1}^{2}}{B_{4}+2\left|B_{3}\right|}, \quad t \in\left[t_{0}, 1\right], s \in[0,1] .
$$

Thus, from (8), (9), (11)-(13), inequality (7) follows.

Now we show that inequality (7) is sharp.

(1) When $B_{1} \geq B_{4}+2\left|B_{3}\right|$, equality holds in (7) for $p(z)=(1+z) /(1-z)$. 
(2) When $B_{1}^{2} \leq 2\left|B_{3}\right|\left(B_{4}+2\left|B_{3}\right|\right)$, we note that $B_{3}$ cannot be a zero, since $B_{1} \geq 0$. Now, consider $p \in \mathcal{P}$ defined by

$$
p(z)=\frac{1+\zeta_{1}\left(\zeta_{2}+1\right) z+\zeta_{2} z^{2}}{1+\zeta_{1}\left(\zeta_{2}-1\right) z-\zeta_{2} z^{2}}
$$

where

$$
\zeta_{1}=\sqrt{\frac{2\left|B_{3}\right|}{B_{4}+2\left|B_{3}\right|}} \text { and } \zeta_{2}= \begin{cases}-\frac{\left|B_{3}\right|\left(4 B_{2}+2 B_{3}\right)}{B_{3}\left|4 B_{2}+2 B_{3}\right|}, & \text { if } B_{3} \neq-2 B_{2} \\ 1, & \text { if } B_{3}=-2 B_{2} .\end{cases}
$$

Then $p_{1}=2 \zeta_{1}$ and $p_{2}=2\left(\zeta_{1}^{2}+\left(1-\zeta_{1}^{2}\right) \zeta_{2}\right)$. A computation shows that $\left|B_{2} p_{1}^{2}+B_{3} p_{2}\right|=0$. Thus

$$
\Psi_{-}\left(p_{1}, p_{2}\right)=\left|B_{1} p_{1}\right|-\left|B_{2} p_{1}^{2}+B_{3} p_{2}\right|=2 B_{1} \zeta_{1}=2 B_{1} \sqrt{\frac{2\left|B_{3}\right|}{B_{4}+2\left|B_{3}\right|}} .
$$

(3) When $B_{1}<B_{4}+2\left|B_{3}\right|$ and $B_{1}^{2}>2\left|B_{3}\right|\left(B_{4}+2\left|B_{3}\right|\right)$, we note that $4 B_{2}+2 B_{3} \neq 0$. Consider $p \in \mathcal{P}$ defined by (14) with

$$
\zeta_{1}=\frac{B_{1}}{B_{4}+2\left|B_{3}\right|} \quad \text { and } \quad \zeta_{2}= \begin{cases}-\frac{\left|B_{3}\right|\left(4 B_{2}+2 B_{3}\right)}{B_{3}\left|4 B_{2}+2 B_{3}\right|}, & \text { if } B_{3} \neq 0, \\ 1, & \text { if } B_{3}=0 .\end{cases}
$$

When $B_{3} \neq 0$, calculations give

$$
\begin{aligned}
& \left|B_{2} p_{1}^{2}+B_{3} p_{2}\right|^{2} \\
& =\left|\left(4 B_{2}+2 B_{3}\right) \zeta_{1}^{2}+2 B_{3}\left(1-\zeta_{1}^{2}\right) \zeta_{2}\right|^{2} \\
& =B_{4}^{2} \zeta_{1}^{4}+4 \operatorname{Re}\left\{B_{3}\left(\overline{4 B_{2}+2 B_{3}}\right) \zeta_{1}^{2}\left(1-\zeta_{1}^{2}\right) \zeta_{2}\right\}+4 B_{3}^{2}\left(1-\zeta_{1}^{2}\right)^{2} \\
& =B_{4}^{2} \zeta_{1}^{4}-4 \zeta_{1}^{2}\left(1-\zeta_{1}^{2}\right) B_{4}\left|B_{3}\right|+4 B_{3}^{2}\left(1-\zeta_{1}^{2}\right)^{2} \\
& =\left(B_{4} \zeta_{1}^{2}-2\left|B_{3}\right|\left(1-\zeta_{1}^{2}\right)\right)^{2} \\
& =\left(\frac{B_{1}^{2}}{B_{4}+2\left|B_{3}\right|}-2\left|B_{3}\right|\right)^{2} .
\end{aligned}
$$

Since $B_{1}^{2}>2\left|B_{3}\right|\left(B_{4}+2\left|B_{3}\right|\right)$, we get

$$
\left|B_{2} p_{1}^{2}+B_{3} p_{2}\right|=\frac{B_{1}^{2}}{B_{4}+2\left|B_{3}\right|}-2\left|B_{3}\right|
$$

which implies

$$
\Psi_{-}\left(p_{1}, p_{2}\right)=\left|B_{1} p_{1}\right|-\left|B_{2} p_{1}^{2}+B_{3} p_{2}\right|=2\left|B_{3}\right|+\frac{B_{1}^{2}}{B_{4}+2\left|B_{3}\right|} .
$$

When $B_{3}=0$, note that $\zeta_{1}=B_{1} / B_{4}=B_{1} /\left(4\left|B_{2}\right|\right)$. Therefore

$$
\Psi_{-}\left(p_{1}, p_{2}\right)=B_{1}\left|p_{1}\right|-\left|B_{2}\right|\left|p_{1}\right|^{2}=2 B_{1} \zeta_{1}-4\left|B_{2}\right| \zeta_{1}^{2}=\frac{B_{1}^{2}}{B_{4}}=2\left|B_{3}\right|+\frac{B_{1}^{2}}{B_{4}+2\left|B_{3}\right|} .
$$

Thus, the proof of Proposition 1 is complete.

Before embarking on the following theorems and their proofs, we note that Proposition 1 ensures that the all resulting inequalities are sharp, (see Remark 2 below which gives an example of how to find such extreme functions). 
Remark 1. Taking $B_{1}=1, B_{2}=0$ and $B_{3}=1$ in Proposition 1 , we obtain the sharp inequalities $-\sqrt{2} \leq$ $\left|p_{2}\right|-\left|p_{1}\right| \leq 2$ for $p \in \mathcal{P}$ given as in (5), which poses the question as to what are the sharp upper and lower bounds for $\left|p_{n+1}\right|-\left|p_{n}\right|$ when $n \geq 2$.

Our first two theorems concern $\mathcal{B}_{1}(\alpha, \beta)$ and $\mathcal{B}_{1}^{*}(\alpha, \beta)$, which when $\beta=0$, give sharp upper and lower bounds for $\left|A_{3}\right|-\left|A_{2}\right|$ functions in $\mathcal{S}^{*}(\alpha)$ and $\mathcal{S}^{* *}(\alpha)$, respectively.

Theorem 2. Let $0 \leq \alpha<1$ and $\beta \geq 0$. If $f \in \mathcal{B}_{1}(\alpha, \beta)$ with coefficients of $F$ given by (2), then

$$
\left|A_{3}\right|-\left|A_{2}\right| \leq \begin{cases}\frac{2(1-\alpha)\left[3-\beta^{2}-\alpha\left(6+5 \beta+\beta^{2}\right)\right]}{(1+\beta)^{2}(2+\beta)}, & \text { when } 0 \leq \beta \leq \beta_{0}, 0 \leq \alpha \leq \alpha_{1} \\ \frac{2(1-\alpha)}{2+\beta}, & \text { otherwise, }\end{cases}
$$

and

$$
\left|A_{2}\right|-\left|A_{3}\right| \leq \begin{cases}\frac{2 \sqrt{1-\alpha}}{\sqrt{\beta^{2}+5 \beta+6}}, & \text { when } 0 \leq \alpha \leq \alpha_{2} \\ \frac{2(1-\alpha)}{\sqrt{\alpha\left(6+5 \beta+\beta^{2}\right)+\beta^{2}-\beta-4}}, & \text { otherwise }\end{cases}
$$

where

$$
\beta_{0}=\frac{1}{2}(-1+\sqrt{5}), \quad \alpha_{1}=\frac{2\left(1-\beta-\beta^{2}\right)}{6+5 \beta+\beta^{2}} \quad \text { and } \quad \alpha_{2}=\frac{5+3 \beta}{6+5 \beta+\beta^{2}}
$$

All the inequalities are sharp.

Proof. From the definition of $\mathcal{B}_{1}(\alpha, \beta)$, we can write

$$
\frac{1}{1-\alpha}\left\{f^{\prime}(z)\left(\frac{f(z)}{z}\right)^{\beta-1}-\alpha\right\}=p(z)
$$

for some $p \in \mathcal{P}$, and so equating coefficients gives

$$
a_{2}=\frac{(1-\alpha) p_{1}}{1+\beta} \quad \text { and } \quad a_{3}=\frac{1-\alpha}{2+\beta}\left[\frac{(1-\alpha)(1-\beta)(2+\beta)}{2(1+\beta)^{2}} p_{1}^{2}+p_{2}\right] .
$$

Hence, from (3) we have

$$
\left|A_{3}\right|-\left|A_{2}\right|=\frac{1-\alpha}{2(1+\beta)^{2}(2+\beta)} \Psi_{+}\left(p_{1}, p_{2}\right),
$$

where, $\Psi_{+}\left(p_{1}, p_{2}\right)$ is given by (1) with

$$
B_{1}=2(1+\beta)(2+\beta), \quad B_{2}=(1-\alpha)(\beta+2)(\beta+3) \quad \text { and } \quad B_{3}=-2(1+\beta)^{2} .
$$

First, we assume that

$$
0 \leq \beta \leq \beta_{0} \quad \text { and } \quad 0 \leq \alpha \leq \alpha_{1} .
$$

Then, $2 B_{2}+B_{3}=2\left(5+3 \beta-\alpha\left(6+5 \beta+\beta^{2}\right)\right) \geq 0$. Since $B_{3} \leq 0$, we obtain

$$
\left|2 B_{2}+B_{3}\right|-\left|B_{3}\right|-B_{1}=2 B_{2}+2 B_{3}-B_{1}=-2\left[2\left(-1+\beta+\beta^{2}\right)+\alpha\left(6+5 \beta+\beta^{2}\right)\right] \geq 0 .
$$

By Proposition 1,

$$
\Psi_{+}\left(p_{1}, p_{2}\right) \leq\left|4 B_{2}+2 B_{3}\right|-2 B_{1}=4 B_{2}+2 B_{3}-2 B_{1}=4\left[3-\beta^{2}-\alpha\left(6+5 \beta+\beta^{2}\right)\right],
$$


which together with (17) establishes the first inequality in (15).

Next, we show that $\left|2 B_{2}+B_{3}\right|<\left|B_{3}\right|+B_{1}$ when $\alpha$ and $\beta$ do not satisfy (19), which from Proposition 1 implies that $\Psi_{+}\left(p_{1}, p_{2}\right) \leq 2\left|B_{3}\right|=4(1+\beta)^{2}$, and so together with (17), establishes the second inequality in (15).

We note that $\alpha>2\left(1-\beta-\beta^{2}\right) /\left(6+5 \beta+\beta^{2}\right)$. If $\alpha \leq(5+3 \beta) /\left(6+5 \beta+\beta^{2}\right)$, then $2 B_{2}+$ $B_{3} \geq 0$, and

$$
\left|2 B_{2}+B_{3}\right|-\left|B_{3}\right|-B_{1}=2 B_{2}+2 B_{3}-B_{1}=-2\left[2\left(-1+\beta+\beta^{2}\right)+\alpha\left(6+5 \beta+\beta^{2}\right)\right]<0 .
$$

If $(5+3 \beta) /\left(6+5 \beta+\beta^{2}\right) \leq \alpha$, then $2 B_{2}+B_{3} \leq 0$, which implies that

$$
\left|2 B_{2}+B_{3}\right|-\left|B_{3}\right|-B_{1}=-2 B_{2}-B_{1}<0 .
$$

Now, we consider

$$
\left|A_{2}\right|-\left|A_{3}\right|=\frac{1-\alpha}{2(1+\beta)^{2}(2+\beta)} \Psi_{-}\left(p_{1}, p_{2}\right),
$$

where $\Psi_{-}\left(p_{1}, p_{2}\right)=-\Psi_{+}\left(p_{1}, p_{2}\right)$ with the same $B_{i}$ given in (18). Putting $B_{4}=\left|4 B_{2}+2 B_{3}\right|$, we observe that

$$
2\left|B_{3}\right|\left(B_{4}+2\left|B_{3}\right|\right)-B_{1}^{2}=2\left|B_{3}\right| B_{4}+4 \beta(1+\beta)^{2}(4+3 \beta) .
$$

Since $B_{4} \geq 0$, by (20), we have $2\left|B_{3}\right|\left(B_{4}+2\left|B_{3}\right|\right) \geq B_{1}^{2}$. Hence, Proposition 1 gives

$$
\left|A_{2}\right|-\left|A_{3}\right| \leq \frac{(1-\alpha) B_{1}}{(1+\beta)^{2}(2+\beta)} \sqrt{\frac{2\left|B_{3}\right|}{B_{4}+2\left|B_{3}\right|}}=\frac{2(1-\alpha)}{\sqrt{\left|5+3 \beta-\alpha\left(6+5 \beta+\beta^{2}\right)\right|+(1+\beta)^{2}}},
$$

which completes the proof of Theorem 2.

When $\beta=0$, we deduce

Theorem 3. Let $0 \leq \alpha<1$ and $f \in \mathcal{S}^{*}(\alpha)$, with coefficients of $F$ given by (2). Then

$$
\left|A_{3}\right|-\left|A_{2}\right| \leq \begin{cases}3(1-\alpha)(1-2 \alpha), & \text { when } 0 \leq \alpha \leq \frac{1}{3} \\ 1-\alpha, & \text { when } \frac{1}{3} \leq \alpha<1\end{cases}
$$

and

$$
\left|A_{2}\right|-\left|A_{3}\right| \leq \begin{cases}\sqrt{\frac{2}{3}} \sqrt{1-\alpha}, & \text { when } 0 \leq \alpha \leq \frac{5}{6} \\ \frac{\sqrt{2}(1-\alpha)}{\sqrt{3 \alpha-2}}, & \text { when } \frac{5}{6} \leq \alpha<1 .\end{cases}
$$

All the inequalities are sharp.

Our next result concerns functions in $\mathcal{B}_{1}^{*}(\alpha, \beta)$, which when $\beta=0$, provides sharp upper and lower bounds for $\left|A_{3}\right|-\left|A_{2}\right|$ for functions in $\mathcal{S}^{* *}(\alpha)$.

Theorem 4. Let $0<\alpha \leq 1$ and $\beta \geq 0$. If $f \in \mathcal{B}_{1}^{*}(\alpha, \beta)$ with coefficients of $F$ given by (2), then

$$
\left|A_{3}\right|-\left|A_{2}\right| \leq \begin{cases}\frac{2 \alpha\left[\alpha(5+3 \beta)-\left(2+3 \beta+\beta^{2}\right)\right]}{(1+\beta)^{2}(2+\beta)}, & \text { when } 0 \leq \beta \leq \beta_{0}, \alpha_{3} \leq \alpha \leq 1, \\ \frac{2 \alpha}{2+\beta^{\prime}}, & \text { otherwise }\end{cases}
$$


and

$$
\left|A_{2}\right|-\left|A_{3}\right| \leq \frac{2 \alpha}{\sqrt{(1+\beta)^{2}+\alpha(5+3 \beta)}}
$$

where

$$
\beta_{0}=\frac{1}{2}(-1+\sqrt{5}) \text { and } \alpha_{3}=\frac{3+5 \beta+2 \beta^{2}}{5+3 \beta} .
$$

All the inequalities are sharp.

Proof. Since

$$
f^{\prime}(z)\left(\frac{f(z)}{z}\right)^{\beta-1}=p(z)^{\alpha},
$$

for some $p \in \mathcal{P}$, equating coefficients gives

$$
a_{2}=\frac{\alpha p_{1}}{1+\beta} \quad \text { and } \quad a_{3}=\frac{\alpha\left[\left(\alpha(3+\beta)-(1+\beta)^{2}\right) p_{1}^{2}+2(1+\beta)^{2} p_{2}\right]}{2(1+\beta)^{2}(2+\beta)} .
$$

Hence, using (3), we have

$$
\left|A_{3}\right|-\left|A_{2}\right|=\frac{\alpha}{2(1+\beta)^{2}(2+\beta)} \Psi_{+}\left(p_{1}, p_{2}\right),
$$

where $\Psi_{+}\left(p_{1}, p_{2}\right)$ is given by (1) with

$$
B_{1}=2(1+\beta)(2+\beta), \quad B_{2}=(1+\beta)^{2}+\alpha(5+3 \beta) \quad \text { and } \quad B_{3}=-2(1+\beta)^{2} .
$$

We note that $2 B_{2}+B_{3}=2 \alpha(5+3 \beta)>0$.

First, we assume that

$$
0 \leq \beta \leq \beta_{0} \quad \text { and } \quad \alpha_{3} \leq \alpha \leq 1
$$

Then, we have

$$
\left|2 B_{2}+B_{3}\right|-\left|B_{3}\right|-B_{1}=2 B_{2}+2 B_{3}-B_{1}=-2\left[3+5 \beta+2 \beta^{2}-\alpha(5+3 \beta)\right] \geq 0 .
$$

By Proposition 1,

$$
\Psi_{+}\left(p_{1}, p_{2}\right) \leq\left|4 B_{2}+2 B_{3}\right|-2 B_{1}=4 B_{2}+2 B_{3}-2 B_{1}=4\left[\alpha(5+3 \beta)-\left(2+3 \beta+\beta^{2}\right)\right],
$$

which from (25) gives the first inequality in (23).

If $\alpha$ and $\beta$ do not satisfy (27), $\alpha<\left(3+5 \beta+2 \beta^{2}\right) /(5+3 \beta)$. Since $2 B_{2}+B_{3}>0$, we have

$$
\left|2 B_{2}+B_{3}\right|-\left|B_{3}\right|-B_{1}=-2\left[3+5 \beta+2 \beta^{2}-\alpha(5+3 \beta)\right]<0 .
$$

By Proposition 1, it follows that

$$
\Psi_{+}\left(p_{1}, p_{2}\right) \leq 2\left|B_{3}\right|=4(1+\beta)^{2} .
$$

From (25), (28) and (29), we obtain the second inequality in (23) as required.

We now consider the lower bound. Then

$$
\left|A_{2}\right|-\left|A_{3}\right|=\frac{\alpha}{2(1+\beta)^{2}(2+\beta)} \Psi_{-}\left(p_{1}, p_{2}\right),
$$

where $\Psi_{-}\left(p_{1}, p_{2}\right)=-\Psi_{+}\left(p_{1}, p_{2}\right)$ with the same $B_{i}$ given in (26). Since $B_{4}=\left|4 B_{2}+2 B_{3}\right| \geq 0$, we have

$$
2\left|B_{3}\right|\left(B_{4}+2\left|B_{3}\right|\right)-B_{1}^{2}=2\left|B_{3}\right| B_{4}+4 \beta(1+\beta)^{2}(4+3 \beta) \geq 0 .
$$


Hence, (30) and Proposition 1 gives

$$
\left|A_{2}\right|-\left|A_{3}\right| \leq \frac{\alpha B_{1}}{(1+\beta)^{2}(2+\beta)} \sqrt{\frac{2\left|B_{3}\right|}{B_{4}+2\left|B_{3}\right|}}=\frac{2 \alpha}{\sqrt{(1+\beta)^{2}+\alpha(5+3 \beta)}}
$$

which completes the proof of Theorem 4 .

When $\beta=0$, we deduce

Theorem 5. Let $0<\alpha \leq 1$ and $f \in \mathcal{S}^{* *}(\alpha)$, with coefficients of $F$ given by (2). Then

$$
\left|A_{3}\right|-\left|A_{2}\right| \leq \begin{cases}\alpha, & \text { when } 0<\alpha \leq 3 / 5 \\ \alpha(5 \alpha-2), & \text { when } 3 / 5 \leq \alpha \leq 1\end{cases}
$$

and

$$
\left|A_{2}\right|-\left|A_{3}\right| \leq \frac{2 \alpha}{\sqrt{5 \alpha+1}} \quad \text { when } 0<\alpha \leq 1 .
$$

All the inequalities are sharp.

At this point, we note that taking $\beta=1$ in Definitions 7 and 8 , defines two classes of functions with bounded turning, which from Theorems 2 and 4, immediately give the following theorem.

Theorem 6. Let $f \in \mathcal{A}$, with coefficients of $F$ given by (2). If $\operatorname{Re} f^{\prime}(z)>\alpha$ for $0 \leq \alpha<1$ and $z \in \mathbb{D}$, then

$$
\left|A_{3}\right|-\left|A_{2}\right| \leq \frac{2(1-\alpha)}{3},
$$

and

$$
\left|A_{2}\right|-\left|A_{3}\right| \leq \begin{cases}\sqrt{\frac{1-\alpha}{3}} & \text { when } 0 \leq \alpha \leq \frac{2}{3} \\ \frac{1-\alpha}{\sqrt{3 \alpha-1}} & \text { when } \frac{2}{3} \leq \alpha<1\end{cases}
$$

Also if $\left|\arg f^{\prime}(z)\right| \leq \alpha \pi / 2$ for $0<\alpha \leq 1$ and $z \in \mathbb{D}$, then

$$
\left|A_{3}\right|-\left|A_{2}\right| \leq \frac{2 \alpha}{3}
$$

and

$$
\left|A_{2}\right|-\left|A_{3}\right| \leq \frac{\alpha}{\sqrt{1+2 \alpha}}
$$

All the inequalities are sharp.

We now consider the classes $\mathcal{C}(\alpha)$ and $\mathcal{C}^{*}(\alpha)$ of convex functions.

Theorem 7. Let $0 \leq \alpha<1$ and $f \in \mathcal{C}(\alpha)$, with coefficients of $F$ given by (2). Then

$$
\left|A_{3}\right|-\left|A_{2}\right| \leq \frac{1}{3}(1-\alpha), \quad \text { when } \quad 0 \leq \alpha<1 \text {, }
$$


and

$$
\left|A_{2}\right|-\left|A_{3}\right| \leq \begin{cases}\frac{1}{2} \sqrt{1-\alpha}, & \text { when } 0 \leq \alpha \leq \frac{7}{16} \\ \frac{1}{48}(25-16 \alpha), & \text { when } \frac{7}{16} \leq \alpha \leq \frac{5}{8} \\ \frac{4}{3} \alpha(1-\alpha), & \text { when } \frac{5}{8} \leq \alpha \leq \frac{3}{4} \\ \frac{2}{3}(1-\alpha)(3-2 \alpha), & \text { when } \frac{3}{4} \leq \alpha \leq \frac{7}{8} \\ \frac{(1-\alpha)(16 \alpha+1)}{24(2 \alpha-1)}, & \text { when } \frac{7}{8} \leq \alpha \leq 1 .\end{cases}
$$

All the inequalities are sharp.

Proof. Since $f \in \mathcal{C}(\alpha)$ if, and only if, $z f^{\prime} \in \mathcal{S}^{*}(\alpha)$, taking $\beta=0$ in (16), for some $p \in \mathcal{P}$ of the form (5) we have

$$
a_{2}=\frac{1}{2}(1-\alpha) p_{1} \quad \text { and } \quad a_{3}=\frac{1}{6}(1-\alpha)\left[(1-\alpha) p_{1}^{2}+p_{2}\right],
$$

which implies from (3) that

$$
\left|A_{3}\right|-\left|A_{2}\right|=\frac{1-\alpha}{6} \Psi_{+}\left(p_{1}, p_{2}\right) \quad \text { and } \quad\left|A_{2}\right|-\left|A_{3}\right|=\frac{1-\alpha}{6} \Psi_{-}\left(p_{1}, p_{2}\right),
$$

where $\Psi_{+}\left(p_{1}, p_{2}\right)$ is given by (1) with $B_{1}=3, B_{2}=2(1-\alpha)$ and $B_{3}=-1$, and $\Psi_{-}\left(p_{1}, p_{2}\right)=$ $-\Psi_{+}\left(p_{1}, p_{2}\right)$. By applying Proposition 1 , we obtain the sharp inequalities (31) and (32).

Theorem 8. Let $0 \leq \alpha<1$ and $f \in \mathcal{C}^{* *}(\alpha)$, with coefficients of $F$ given by (2). Then

$$
\left|A_{3}\right|-\left|A_{2}\right| \leq \frac{\alpha}{3}, \quad \text { when } 0<\alpha \leq 1,
$$

and

$$
\left|A_{2}\right|-\left|A_{3}\right| \leq \begin{cases}\alpha(1-\alpha), & \text { when } 0<\alpha \leq \frac{1}{6} \\ \frac{\alpha(12 \alpha+13)}{12(3 \alpha+1)}, & \text { when } \frac{1}{6} \leq \alpha \leq \frac{5}{12} \\ \frac{\alpha}{\sqrt{3 \alpha+1}}, & \text { when } \frac{5}{12} \leq \alpha \leq 1 .\end{cases}
$$

All the inequalities are sharp.

Proof. Since $f \in \mathcal{C}^{*}(\alpha)$ if, and only if, $z f^{\prime} \in \mathcal{S}^{* *}(\alpha)$, taking $\beta=0$ in (24), for some $p \in \mathcal{P}$ of the form (5) we have

$$
a_{2}=\frac{1}{2} \alpha p_{1} \quad \text { and } \quad a_{3}=\frac{1}{12} \alpha\left[(3 \alpha-1) p_{1}^{2}+2 p_{2}\right],
$$

which implies from (3) that

$$
\left|A_{3}\right|-\left|A_{2}\right|=\frac{1-\alpha}{12} \Psi_{+}\left(p_{1}, p_{2}\right) \quad \text { and } \quad\left|A_{2}\right|-\left|A_{3}\right|=\frac{1-\alpha}{12} \Psi_{-}\left(p_{1}, p_{2}\right),
$$


where $\Psi_{+}\left(p_{1}, p_{2}\right)$ is given by (1) with $B_{1}=6, B_{2}=3 \alpha+1$ and $B_{3}=-2$, and $\Psi_{-}\left(p_{1}, p_{2}\right)=$ $-\Psi_{+}\left(p_{1}, p_{2}\right)$. By applying Proposition 1 , we obtain the sharp inequalities (33) and (34).

\section{Finding Extreme Functions}

Proposition 1 tells us that all the inequalities in the above theorems are sharp. In order to illustrate how to calculate extreme functions, we find the extreme functions for the inequalities in Theorem 3 , noting that similar calculations will find extreme functions for all the theorems presented in the paper.

We first discuss the sharpness of (21). Note first that for this class, we have

Since $f \in \mathcal{S}^{*}(\alpha)$, from (4), we can write

$$
z f^{\prime}(z)=f(z)(\alpha+(1-\alpha) p(z))
$$

for some $p \in \mathcal{P}$. Let $p$ be of the form (5). Equating coefficients in (35) gives

$$
a_{2}=(1-\alpha) p_{1} \quad \text { and } \quad a_{3}=\frac{1}{2}(1-\alpha)\left[(1-\alpha) p_{1}^{2}+p_{2}\right] .
$$

Using (3) we obtain

$$
\left|A_{2}\right|=(1-\alpha)\left|p_{1}\right| \quad \text { and } \quad\left|A_{3}\right|=\frac{(1-\alpha)}{2}\left|\left(3(1-\alpha) p_{1}^{2}-p_{2}\right)\right| .
$$

Thus

$$
\left|A_{3}\right|-\left|A_{2}\right|=\frac{1-\alpha}{2} \Psi_{+}\left(p_{1}, p_{2}\right) \text { and }\left|A_{2}\right|-\left|A_{3}\right|=\frac{1-\alpha}{2} \Psi_{-}\left(p_{1}, p_{2}\right),
$$

where $\Psi_{+}\left(p_{1}, p_{2}\right)$ is given by (1) with $B_{1}=2, B_{2}=3(1-\alpha)$ and $B_{3}=-1$, and $\Psi_{-}\left(p_{1}, p_{2}\right)=$ $-\Psi_{+}\left(p_{1}, p_{2}\right)$.

When $0 \leq \alpha \leq 1 / 3$, since $\left|2 B_{2}+B_{3}\right| \geq\left|B_{3}\right|+B_{1}$, equality holds on choosing $p(z)=(1+$ $z) /(1-z)$ in (35). Moreover, taking $p_{1}=2$ and $p_{2}=2$ in (36), we have $\left|A_{2}\right|=2(1-\alpha)$ and $\left|A_{3}\right|=(1-\alpha)(5-6 \alpha)$. When $1 / 3 \leq \alpha<1$, equality holds on choosing $p(z)=\left(1+z^{2}\right) /\left(1-z^{2}\right)$ in (35). In this case, $p_{1}=0$ and $p_{2}=2$, and so we obtain $A_{2}=0$ and $\left|A_{3}\right|=1-\alpha$.

We finally discuss the sharpness of (22). Recall that $2\left|B_{3}\right|\left(B_{4}+2\left|B_{3}\right|\right) \geq B_{1}^{2}$, where $B_{4}=\mid 4 B_{2}+$ $2 B_{3} \mid$ for $0 \leq \alpha<1$.

If $\alpha=5 / 6$, then $4 B_{2}+2 B_{3}=0$, and if $0 \leq \alpha<5 / 6$, then $4 B_{2}+2 B_{3}>0$, which gives

$$
\delta:=-\frac{\left|B_{3}\right|\left(4 B_{2}+2 B_{3}\right)}{B_{3}\left|4 B_{2}+2 B_{3}\right|}=1
$$

Using the same argument as in (2) in the proof of Proposition 1, an extreme function $f$ is obtained from (35) with $p(z)=\left(1+2 t z+z^{2}\right) /\left(1-z^{2}\right)$ and $t=1 /(\sqrt{6} \sqrt{1-\alpha})$.

This gives $p_{1}=2 t$ and $p_{2}=2$, and so $\left|A_{2}\right|=\sqrt{2 / 3} \sqrt{1-\alpha}$ and $A_{3}=0$. If $5 / 6<\alpha<1$, then $4 B_{2}+2 B_{3}<0$, which gives $\delta=-1$. Again same argument as in (2) in the proof of Proposition 1 shows that an extreme function $f$ is obtained by (35), with $p(z)=\left(1-z^{2}\right) /\left(1-2 t z+z^{2}\right)$ and $t=1 /(\sqrt{2} \sqrt{3 \alpha-2})$. Thus, $p_{1}=2 t$ and $p_{2}=-2+4 t^{2}$, which implies that $\left|A_{2}\right|=\sqrt{2}(1-\alpha) / \sqrt{3 \alpha-2}$ and $A_{3}=0$.

Remark 2. We note that when $f \in \mathcal{C}^{* *}(\alpha)$, the upper and lower bounds for $\left|A_{3}\right|-\left|A_{2}\right|$ in Theorem 8 are identical to those obtained for $\left|a_{3}\right|-\left|a_{2}\right|$ in [9], thus giving another example of the invariance of coefficient and functionals for some classes of convex functions, as demonstrated in [13].

Remark 3. We finally remark that in [9] individual proofs concerning upper and lower bounds for the difference $\left|a_{3}\right|-\left|a_{2}\right|$ were given. These can be more easily obtained using Proposition 1. 
Remark 4. Although we have not discussed the class $\mathcal{K}$ of close-to-convex functions in this paper, i.e., functions $f \in \mathcal{A}$ with $g \in \mathcal{S}^{*}$ satisfying

$$
\operatorname{Re} \frac{z f^{\prime}(z)}{g(z)}>0 \quad \text { for } z \in \mathbb{D}
$$

the question arises as to whether the lower bound $-1 \leq\left|A_{3}\right|-\left|A_{2}\right|$ proved in Theorem 1 for $f \in \mathcal{S}$ can be improved for $f \in \mathcal{K}$.

Using Lemma 2, and assuming that $g(z)$ is the Koebe function and that the coefficient $a_{2} \geq 0$, it is not too difficult to show that this lower bound can be increased to $-2 / 3$, and this lower bound is the best possible. On the basis of this, we make the following conjecture.

Conjecture Let $f \in \mathcal{K}$, with inverse coefficients given by (2), then $-2 / 3 \leq\left|A_{3}\right|-\left|A_{2}\right| \leq 3$.

Author Contributions: Both authors have equal contributions. Both authors have read and agreed to the published version of the manuscript.

Funding: This work was partially supported by the National Research Foundation of Korea (NRF) grant funded by the Korea government (MSIP; Ministry of Science, ICT \& Future Planning) (No. NRF-2017R1C1B5076778).

Conflicts of Interest: The authors declare no conflict of interest.

\section{References}

1. De Branges, L. A proof of the Bieberbach conjecture. Acta Math. 1985, 154, 137-152. [CrossRef]

2. Duren, P.L. Univalent Functions; Springer: Berlin/Heidelberg, Germany, 1983.

3. Hayman, W.K. On successive coefficients of univalent functions. J. Lond. Math. Soc. 1963, 38, $228-243$. [CrossRef]

4. Grinspan, A.Z. The sharpening of the difference of the moduli of adjacent coefficients of schlicht functions. In Problems in Modern Function Theory, Proceedings of the Conference on Modern Problems of the Geometric Theory of Functions; Institute of Mathematics, Siberian Branch, Academy of Sciences of the USSR: Novosibirsk, Russia, 1976; pp. 41-45.

5. Hayman, W.K. Multivalent Functions, 2nd ed.; Cambridge University Press; New York, NY, USA, 1994.

6. Ilina, L.P. The relative growth of nearby coefficients of schlicht functions. Mat. Zametki 1968, 4, 715-722.

7. Obradović, M.; Thomas, D.K.; Tuneski, N. On the difference of coefficients of univalent functions. arXiv 2020, arXiv:2004.06369v.

8. Löwner, C. Untersuchungen uber schlichte konforme Abbildungen des Einheitskreises. I. Math. Ann. 1923, 89, 103-121.

9. Sim, Y.J.; Thomas, D.K. On the difference of coefficients of starlike and convex functions. Mathematics 2020, 8, 1521. [CrossRef]

10. Leung, Y. Successive coefficients of starlike functions. Bull. Lond. Math. Soc. 1978, 10, 193-196. [CrossRef]

11. Cho, N.E.; Kowalczyk, B.; Lecko, A. The sharp bounds of some coefficient functionals over the class of functions convex in the direction of the imaginary axis. Bull. Aust. Math. Soc. 2019, 100, 86-96. [CrossRef]

12. Libera, R.J.; Złotkiewicz, E.J. Coefficient bounds for the inverse of a function with derivatives in $\mathcal{P}$. Proc. Am. Math. Soc. 1983, 87, 251-257. [CrossRef]

13. Thomas, D.K.; Verma, S. Invariance of the coefficients of strongly convex functions. Bull. Aust. Math. Soc. 2017, 95, 435-445.

Publisher's Note: MDPI stays neutral with regard to jurisdictional claims in published maps and institutional affiliations.

(C) 2020 by the authors. Licensee MDPI, Basel, Switzerland. This article is an open access article distributed under the terms and conditions of the Creative Commons Attribution (CC BY) license (http://creativecommons.org/licenses/by/4.0/). 